\title{
HORARIOS DE OCURRENCIA DE PARTOS EN CABRAS DEL VALLE DE MÉXICO (CAPRA HIRCUS)\#
}

\author{
TIME OF PARTURITION IN GOATS AT THE VALLEY OF MEXICO (CAPRA HIRCUS)
}

\author{
Sampson, J.J. ${ }^{1}$, Ducoing, A.E. ${ }^{1}$ y Álvarez, L. ${ }^{1 *}$
}

${ }^{1}$ Universidad Nacional Autónoma de México. Facultad de Medicina Veterinaria y Zootecnia. Departamento de Producción Animal. Ciudad de México. México. *alorenzo@servidor.unam.mx

\author{
Palabras ClaVe ADICIONALES \\ Conducta materna. Época de partos.
}

\section{RESUMEN}

Con el objetivo de determinar el patrón en los horarios de ocurrencia de partos en la cabra doméstica del valle de México, se evaluó la información en registros de 832 nacimientos de 1991 a 1997 y del año 2000 al 2007. En la evaluación de la información se consideraron horarios diurnos (07:00-19:00 h) y nocturnos (19:00-07:00 h). Se utilizaron tablas de contingencia y modelos lineales, considerando la raza, el tipo de parto, el sexo y peso de las crías, así como la estación del año. La distribución de los partos durante el día no fue homogénea $(p<0,01)$ y el $82 \%$ de ellos se concentró en horarios diurnos, con un claro pico $(31,4 \%)$ entre las 11:00 y las 13:00 h. El horario de los nacimientos no se asoció con la raza de la madre, el tipo de parto, el peso de las crías, el sexo de la cría ni la estación del año $(p>0,05)$. Se concluye que, en el valle de México, la mayoría de los partos $(>80 \%)$ en la cabra ocurre en horarios diurnos, con una mayor incidencia entre las 11:00 y las 13:00 h.

\section{SUMMARY}

With the aim of determining the time pattern in parturition in the domestic goat in the valley of Mexico, the information of 832 births from 1991 to 1997 and 2000 to 2007 was evaluated. Diurnal (07:00-19:00 h) and nocturnal times (19:00-07:00 h) were considered in analysis. Tables of

\#El trabajo formó parte de la tesis de licenciatura del primer autor. Universidad Nacional Autónoma de México.

Recibido: 23-9-09. Aceptado: 22-10-09.

\section{AdDitional KEYWORDS}

Maternal behavior. Birth season.

contingency and linear models were used, considering the breed, type of parturition, sex and weight of the kid and the season of the year. Distribution of parturition during the day was not homogenous $(p<0.01)$. A high percentage of them $(82 \%)$ were concentrated in diurnal times, with a clear peak $(31 \%)$ between 11:00 and 13:00 h. Time of birth was neither associated with the breed of the mother, type of parturition, weight of the kid, sex of the young nor the season of the year $(p>0.05)$. It is concluded that, in the valley of Mexico, the most of the goats' births (>80\%) occurs during diurnal times, with a greater incidence between 11:00 and 13:00 h.

\section{INTRODUCCIÓN}

En varias especies de mamíferos se han identificado al menos dos tipos de pautas para la distribución en los horarios en que ocurren los partos. En el primero, algunas especies tienden a parir en diferentes periodos a lo largo del día, sin tener alguna predilección aparente por un horario en particular como es el caso de la oveja Awassi en Iraq (Younis y El-Gaboory, 1978) en el segundo, los partos tienden a concentrarse en horarios específicos de la noche como se ha visto en equinos (Rossdale y Short, 1967) $y$ en ratas (Bosc, 1987).

En algunas razas de cabras como la Alpina Francesa o sus cruzas con la raza Saanen (Bosc et al., 1988; Lickliter, 1985), y

Arch. Zootec. 61 (234): 297-300. 2012. 
en ovejas de la raza Dorset Horn y Merino (George, 1969), se han identificado diferentes patrones de distribución y ocurrencia de los horarios de parto. En ambas especies se ha encontrado una preferencia por horarios diurnos, aunque en ovejas se ha registrado también una tendencia hacia una distribución más homogénea durante el día (Martínez, 2005) y ligeramente diferente en algunas razas (George, 1969). En cabras por su parte, se ha visto que los nacimientos tienden a concentrarse al medio día, de forma independiente a la raza (Bosc et al., 1988; Lickliter, 1985) y en menor proporción durante la tarde y noche (Romano y Piaggio, 1999). Así, basándose en los estudios disponibles hasta el momento y realizados en geografías diferentes, la cabra parece pertenecer al grupo cuyo parto ocurre en horarios definidos del día y principalmente durante periodos de luz. No existen informes disponibles del tema originados en México.

El conocimiento de los horarios del día en que ocurre la mayor frecuencia de los partos puede ayudar a identificar estrategias de tipo evolutivo que permitan un mayor éxito a las especies. Además, en las unidades de producción caprina, la supervisión de las hembras al momento del parto facilitaría la atención adecuada y una oportuna intervención en casos de distocia o nacimiento de cabritos débiles.

En el presente estudio se analizó la información de los horarios de parto en 832 nacimientos, en busca de determinar el patrón característico en la cabra doméstica criada en intensivo en el valle de México.

\section{MATERIAL Y MÉTODOS}

Se analizó la información de 832 partos ocurridos entre 1991 a 1997 y del año 2000 al 2007. La información se obtuvo de registros en el Centro de Enseñanza Práctica e Investigación en Producción y Salud Animal (CEPIPSA) de la Facultad de Medicina Veterinaria y Zootecnia de la Universidad Nacional Autónoma de México. El centro está ubicado a $2760 \mathrm{msnm}$, a $19^{\circ} 13^{\prime} \mathrm{N}, 99^{\circ}$ $8^{\prime} \mathrm{W}$, en la Ciudad de México. Durante el periodo de registro, las cabras fueron manejadas en condiciones intensivas, de forma estabulada las 24 horas, recibiendo alimentación tres veces por día (08:00 h, 12:00 hy 14:00 h) a base de heno de alfalfa, avena, ensilado de maíz y concentrado. La disponibilidad de agua fue siempre a libre acceso. Los animales fueron expuestos al fotoperiodo natural de la zona y su ubicación siempre fue la misma. En las épocas de nacimiento, las cabras fueron vigiladas las $24 \mathrm{~h}$ del día para el registro de la información y para el auxilio en la labor de parto si era necesario. En partos dobles, triples o cuádruples se consideró únicamente la hora de expulsión de la primera cría; para determinar la interacción entre el sexo de la cría y el horario del parto, se consideró también sólo a la primera cría. Para el análisis del porcentaje de partos, la estación del año y el sexo de la cría, el horario se dividió en periodos de 8 fracciones durante el día, a partir de la 01:00-04:00 h, 04:00-07:00 h, 07:00-10:00 h, 10:00-13:00 h, 13:00-16:00 h, 16:00-19:00 h, 19:00-22:00 h y 22:00-01:00 h. Los periodos 07:00-19:00 h, fueron considerados como periodos diurnos, y el resto del día (19:0007:00 h) como periodo nocturno. En el análisis se consideró el año y la estación como posibles efectos sobre la hora del parto. La información se analizó mediante un análisis para tablas de contingencia y un modelo lineal, con el uso del paquete estadístico JMP versión 5.1 (SAS, 1999). En el modelo se consideró a la hora del parto como variable dependiente y al año de estudio, con la estación del año anidada dentro, al sexo de la cría, a la raza de la madre y al tipo de parto como variables explicativas.

\section{RESULTADOS}

La distribución de los horarios de parto no fue homogénea durante el día $(p<0,01)$. El $82 \%$ de los partos ocurrió en horarios diurnos (07:00-19:00 h; figura 1), con un pico 


\section{HORARIOS DE PARTO EN CABRAS}

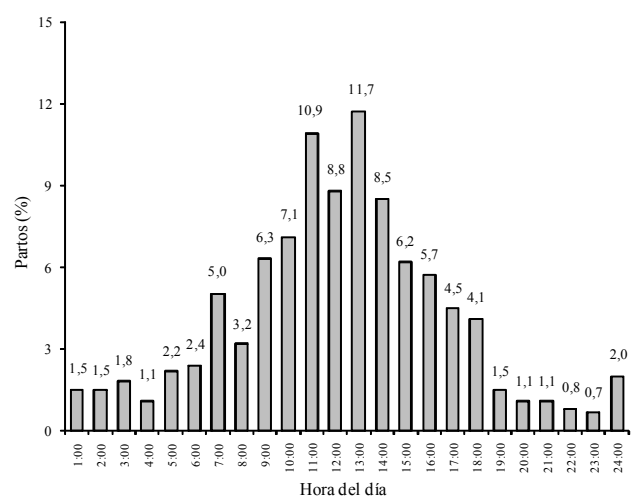

Figura 1. Distribución de los horarios de parto a lo largo del día en el total $(n=832)$ de nacimientos registrados. (Distribution of parturitions throughout the day).

claramente identificable entre las 11:00 y 13:00 horas.

No se encontró un efecto del año sobre el horario de parto $(\mathrm{p}>0,05)$.

Del total de partos con información (832), 387 ocurrieron en primavera $(46,4 \%), 63$ en verano $(7,6 \%), 148$ en otoño $(17,8 \%)$ y 234 en invierno $(28,1 \%)$. La estación del año no tuvo un efecto significativo sobre el horario del parto durante el día $(\mathrm{p}>0,05)$.

No se encontró un efecto significativo del sexo de la cría, de la raza de la madre, del peso de las crías, del tipo de parto, ni del número de las crías sobre el horario de parto $(\mathrm{p}>0,05)$.

\section{DISCUSIÓN}

Aunque se presentaron partos durante todo el día, su distribución no fue homogénea y mostró un patrón claramente diurno, con el $82 \%$ de ellos ocurriendo entre las 07:00 y 19:00 h. La tendencia no cambió en ninguna de las razas representadas, lo que sugiere que se trata de una estrategia fuertemente establecida en la especie. Se sabe que el efecto de las condiciones climáticas ambientales sobre la supervivencia de las crías recién nacidas es muy importante; los corderos nacidos por la tarde en zonas cálidas tienen una mayor oportunidad de so- brevivir que aquellos nacidos por la mañana (McDonald, 1961). Es ventajoso, por lo tanto, que algunas especies utilicen estrategias que les permitan ajustarse a dichas condiciones, asegurando con ello la viabilidad de sus crías.

El proceso que determinará el horario en que ocurrirá el parto en cabras podría estar regulado por eventos de naturaleza circadiana, como el fotoperiodo y temperatura ambiental (Piccione y Caola, 2002). En ratas (Lincoln y Porter 1976) y alpacas (Knight et al., 1995) un alto porcentaje de los nacimientos está regulado por el fotoperiodo. La glándula pineal y su principal secreción, la melatonina, parecen ser los mediadores que permiten al fotoperiodo regular la hora del nacimiento en los roedores (Bosc, 1987) y rumiantes (Apostolakis et al., 1993). En humanos se han encontrado receptores para la melatonina en el miometrio, y la hormona puede modular la función del músculo uterino (Schlabritz-Loutsevitch et al., 2003). Aplicada in vitro, la melatonina actúa sinérgicamente con la oxitocina para promover la contracción muscular (Sharkey et al., 2009). Además, en conejos la sensibilidad del útero a la oxitocina cambia durante el día en fluctuaciones provocadas por el fotoperiodo (Ninomiya-Alarcon et al., 2004).

En la determinación de los periodos más importantes a vigilar durante el día, la información obtenida resulta de utilidad para racionar recursos y personal. En las granjas comerciales, una vigilancia especial durante la época de nacimientos permite supervisar que el parto ocurra sin riesgos para la madre y la cría; ello mejorará las probabilidades de supervivencia de las crías y contribuirá a incrementar la eficiencia de la granja. Se concluye que, en el valle de México, la mayoría de los partos de cabras ( $82 \%$ ) ocurre en horarios diurnos, con una mayor incidencia entre las 11:00 y las 13:00 h del día y que dicha tendencia no es afectada por la estación del año, el peso de la cría, el sexo de la cría, el tipo de parto ni la raza de la madre. 


\section{SAMPSON, DUCOING Y ÁLVAREZ}

\section{AGRADECIMIENTOS}

Se agradece a todo el personal y servicio social del Centro de Enseñanza, Práctica e

\section{BIBLIOGRAFÍA}

Apostolakis, E., Rice, K.E., Longo, L.D., SeronFerre, M. and Yellon, S.M. 1993. Time of day of birth and absence of endocrine and uterine contractile activity rhythms in sheep. $A m \mathrm{~J}$ Physiol-Endoc M, 264: 534-540.

Bosc, M., Guillimin, P., Bourgy, G. and Pignon, P 1988. Hourly distribution of time of parturition in the domestic goat. Theriogenology, 30: 2333.

Bosc, M.J. 1987. Time of parturition in rats after melatonin administration or change of photoperiod. J Reprod Fertil, 80: 563-568.

George, J.M. 1969. Variation in the time of parturition of Merino and Dorset Horn ewes. J Agric Sci, 73: 295-299.

Knight, T.W., Death, A.F. and Wyeth, T.K. 1995. Photoperiodic control of the time of parturition in alpacas (Lama pacos). Anim Reprod Sci, 39: 259-265.

Lickliter, R.E. 1985. Behavior associated with parturition in the domestic goat. Appl Anim Behav Sci, 13: 335-345.

Lincoln, D.W. and Porter, D.G. 1976. Timing of the photoperiod and the hour of birth in rats. Nature, 260: 780-781.

Martínez, D. 2005. Tendencias en la distribución de los horarios de parto en ovejas de diferente raza en el altiplano mexicano. Licenciatura, Benemérita Universidad Autónoma de Puebla, Puebla.

McDonald, I. 1961. Physiological limitations on
Investigación en Producción y Salud Animal (CEPIPSA), por su labor de registro y captura histórica de datos, especialmente a Nicolás y Samuel Andrade.

reproduction in the sheep. Aust Vet J, 37: 99104.

Ninomiya-Alarcon, J.G., Hudson, R., ReyesGuerrero, G., Barrera-Mera, B. and GuevaraGuzman, R. 2004. Effect of photoperiod on the mechanical response of the pregnant rabbit uterus to oxytocin. Am J Physiol-Reg I, 287: R174-180.

Piccione, G. and Caola, G. 2002. Biological rhythm in livestock. J Vet Sci, 3: 145-157.

Romano, J.E. and Piaggio, J. 1999. Time of parturition in Nubian goats. Small Ruminant Res, 33: 285288.

Rossdale, P.D. and Short, R.V. 1967. The time of foaling of Thoroughbred mares. J Reprod Fertil, 13: 341-343.

SAS. 1999. Sas statistics users' guide. Cary, N.C. USA.

Schlabritz-Loutsevitch, N., Hellner, N., Middendorf, R., Muller, D. and Olcese, J. 2003. The human myometrium as a target for melatonin. J Clin Endocr Metab, 88: 908-913.

Sharkey, J.T., Puttaramu, R., Word, R.A. and Olcese, J. 2009. Melatonin synergizes with oxytocin to enhance contractility of human myometrial smooth muscle cells. J Clin Endocr Metab, 94: 421-427.

Younis, A.A. and El-Gaboory, I.A.H. 1978. On the diurnal variation in lambing and time for placenta expulsion in Awassi ewes. J Agric Sci, 91: 757760.

Archivos de zootecnia vol. 61, núm. 234, p. 300. 